\title{
Messaggio di saluto
}

\author{
di Rita Levi Montalcini
}

Un primo incontro con il professore Gian Paolo Cesaretti avvenne nell'Agosto 2006, a Napoli, in occasione di una mia visita all'Università "Parthenope". Da allora, ho saputo della fondazione, da parte sua e della famiglia, di una Fondazione dedicata al ricordo del figlio Simone Cesaretti.

Scopo di questa Fondazione, come mi è stato detto, è quello di aiutare i giovani a superare i momenti difficili della vita, credendo nei valori. Se non si crede nei valori è difficile continuare la vita.

Un caso particolare, del quale si occupa questa Fondazione, si tratta di comunicare ai giovani quanto è importante credere nei valori, credere nell'importanza di questi per potere continuare a vivere. Io sono molto lieta e veramente ringrazio il professore Cesaretti per avermi chiesto di parlare di questo.

Nel mio lungo percorso, io ritengo che la cosa più importante è la fiducia nella vita, la fiducia nell'importanza dei valori. Che si intende per valori? Capire che la vita non ha senso se noi perdiamo tale fiducia, e la fiducia nei valori deriva dalla conoscenza e dalla capacità di vedere che ognuno di noi può contribuire al benessere non soltanto proprio ma di quelli attorno a noi.

Io ritengo splendida la finalità di questa Fondazione, e di questo sono molto grata al professore Cesaretti che mi ha chiesto di parlare dei valori, e non della mia vita, lunghissima e diciamo difficile ma anche fortunata. Io ritengo che soltanto questo $\mathrm{mi}$ ha sostenuto in momenti non facili di persecuzione, in momenti direi veramente tragici. La fiducia e l'ottimismo, in me naturale, mi ha portato in quei momenti a non perdere questa fiducia, a vivere con serenità anche $\mathrm{i}$ momenti difficili.

Ai giovani, ai quali appunto mi rivolgo, qualunque sia la loro età, io desidero mandare questo mio messaggio: la vita vale in quanto crediamo nei valori. Quali valori? Valori etici prima di tutti, valori basati non solo sulla 
conoscenza ma anche sulla cultura. Tutto si può superare se noi crediamo nei valori... anche nei momenti tragici.

Non so quanti di quelli che ascoltano ricordano lo splendido messaggio del grande scrittore Primo Levi. Primo Levi ha vissuto in momenti tragici ad Auschwitz, miracolosamente salvo da questo e, tuttavia, non ha mai perduto questo senso dei valori. Io ho avuto la fortuna e il privilegio di essere sua amica, di vedere in lui uno splendido campione dell'essere umano. A tutti i giovani io vorrei dire leggete i suoi splendidi libri, sono libri che dimostrano come i momenti più tragici, più difficili, possono essere superati se non perdiamo mai la fiducia nei valori e i valori sono culturali, sono cultura di profonda conoscenza e di che cosa siamo noi in questo mondo nel quale, in questo momento, la situazione non è facile. Ma ai giovani posso dire, avendo io stessa passato momenti difficili, che quello che mi ha sempre salvato e protetto è credere enormemente nel senso di questi valori, valori che superano le difficoltà personali, tanto noi siamo parte di un mondo e nella parte di questo mondo noi possiamo vivere anche bene in momenti tragici che possono essere quelli vicini a una morte naturale o non naturale o in momenti di vero sconforto. Ai giovani davanti a me io dico credete in quello, credete nella cultura, credete che quello che rende la vita degna di essere vissuta è questo e lo si può provare sia aiutando il prossimo che superando le difficoltà personali o le difficoltà in momenti anche difficili come quelli che stiamo vivendo.

Come vede a cento anni uno può ancora fare qualcosa, forse perché io credo nell'importanza di impegnarci in pieno, in un modo o nell'altro, nella vita aiutando il prossimo e sviluppando determinati valori. 